\title{
Die Darstellung der katholischen und evangelischen Kirche in der Tagesschau
}

\author{
Hannah Thielmann • Markus Schäfer
}

Eingegangen: 16. Februar 2021 / Angenommen: 4. August 2021 / Online publiziert: 17. September 2021 (C) Der/die Autor(en) 2021

Zusammenfassung Der persönliche Bezug zur Institution Kirche hat in den vergangenen Jahren in der deutschen Bevölkerung und auch bei Journalisten kontinuierlich abgenommen. Gleichzeit bleiben die gesellschaftliche Bedeutung der katholischen und evangelischen Kirche sowie ihr gesetzlich geregeltes Verhältnis zum Rundfunk bestehen. Der vorliegende Beitrag untersucht mithilfe einer quantitativen Inhaltsanalyse von 514 Tagesschau-Beiträgen zu drei Messzeitpunkten (1998/99, 2008/09 und 2018/19) die Berichterstattung über die beiden großen christlichen Kirchen. Im Zentrum stehen dabei die Fragen, in welchen Kontexten bzw. bei welchen Ereignissen über Kirche im Zeitverlauf berichtet und wie positiv oder negativ sie in diesem Zusammenhang dargestellt wird. Es zeigt sich, dass sich die Relevanz der beiden Kirchen in der Tagesschau in den letzten 20 Jahren nicht verändert hat, dass allerdings die katholische Kirche gegenüber der evangelischen Kirche überrepräsentiert ist und signifikant negativer dargestellt wird. Über Ereignisse wird häufiger berichtet als über Stellungnahmen, und die Tendenz der Kirchen-Beiträge ist im Laufe der Jahre signifikant negativer geworden.

Schlüsselwörter Kirche · Religion · Framing · Medienberichterstattung · Inhaltsanalyse

Die empirische Erhebung fand im Rahmen der Bachelorarbeit von Hannah Thielmann am Institut für Publizistik der Johannes Gutenberg-Universität Mainz unter der Erstbetreuung von Dr. Markus Schäfer und der Zweitbetreuung von Prof. Dr. Oliver Quiring statt.

Hannah Thielmann, B.A. $(\bowtie) \cdot$ Dr. Markus Schäfer

Institut für Publizistik, Johannes Gutenberg-Universität Mainz, Jakob-Welder-Weg 12, 55128 Mainz, Deutschland

E-Mail: hthielma@students.uni-mainz.de

Dr. Markus Schäfer

E-Mail: markus.schaefer@uni-mainz.de 


\section{The representation of the Catholic and Protestant churches in the Tagesschau}

Abstract Although the church is still accepted as an institution within the framework of diaconia and caritas in Germany and is largely recognized as a fixed social factor, the personal relationship to the church as an institution has steadily decreased in recent years among the German population in general and also among journalists in particular. There are tendencies towards secularisation in Germany. Hence, it can be assumed that this increasing secularisation in society and journalism has an impact on the setting of content and topics and the evaluation of the Catholic and Protestant churches. Furthermore, it can be assumed that media coverage is the primary (perhaps even only) source of information about the churches for a considerable part of the population. The fewer Germans are active church members, the more likely it becomes that the only information about church comes from the media. Consequently, the image of the church in the media can have a decisive impact on the ideas of the German population about the church. At the same time, the relationship between the Catholic and Protestant Church on one hand and broadcasting (radio and television) on the other is regulated by law, so that, for example, the churches are represented in the supervisory authorities of public broadcasting corporations.

With this knowledge, the question arises whether and to what extent the coverage of the Catholic and Protestant churches has changed in recent years. Due to complex realities, journalists are bound to select information and place emphasis on particular aspects, which means that the perspective on certain topics can be largely determined by journalistic information. Topics are framed this way and portrayed through the lenses of the journalist's image of church. In addition to the journalistic frames, many other factors such as culture, political orientation and the journalistic selfimage determine the creation of these media frames directly and in turn indirectly via the journalist's frames.

However, how the churches are framed in the media coverage has so far hardly been researched. Based on former research, it can only be stated that compared to the Protestant church, the Catholic church is overrepresented in the German media. Furthermore, religion only appears worth reporting if it is polarizing or linked to other topics, e.g. politics. Moreover, the church is being reported on in a personalised manner, and the evaluations of the church as an institution are ambivalent.

In order to narrow this research gap and to examine the reporting on the Catholic and Protestant church over time, a quantitative content analysis of 514 Tagesschau segments was carried out at three points of time (1998/99, 2008/09 and 2018/19). The coverage of the Tagesschau was examined because it is still the news programme with the widest reach in Germany and simultaneously offers an orientation function to other journalists. This approach had been chosen to answer the question, in which contexts or at which events church was reported on over time and how positively or negatively it was presented in the respective contexts.

It turns out that firstly the relevance of the Catholic and Protestant churches in the coverage of the Tagesschau has neither increased nor decreased over the years. This is supported by the fact that neither the number, nor the length or the position of the 
segments about church has changed significantly. Secondly, it can be stated that the Catholic church is overrepresented in the coverage in comparison to the Protestant church, which is in line with previous research results, but runs contrary to the current social distribution in Germany. Thirdly, it is noticeable that the churches are often only reported on in connection with church-specific topics and less with church-independent topics, which has not changed over the years. Besides it is worth mentioning that the number of articles reporting on church events has increased significantly over the years, while church statements have become increasingly rare. Thematically, the image of the churches in the Tagesschau is specifically framed in such a way that most of the segments are about church events, political discussions, and church politics. The image of the large institutions is thus being strengthened and at the same time reduced to internal issues and conflicts. This topic setting has not changed significantly in the last 20 years. However, journalists seem to differentiate between the two denominations, since the Protestant church is significantly more often associated with non-church matters than the Catholic church. Fourth, contrary to other research findings on tabloidisation, no personalisation tendencies in church reporting can be found in the last 20 years. Finally, reporting on the church can be classified as negative and critical. On one hand, this has increased significantly over the years, on the other hand, the reporting on the Catholic church has a significantly more negative tendency than the Protestant church. Moreover, it is worth mentioning that at every point of time the journalists make use of "opportune witnesses", so that negative statements about the church are more frequently quoted than the positive ones. All in all it can be said that the coverage of the Tagesschau about the churches is becoming more and more critical and distanced.

Keywords Church $\cdot$ Religion $\cdot$ Framing $\cdot$ Media coverage $\cdot$ Content analysis

\section{Einleitung}

Kirche hatte selten einen so schweren Stand in der deutschen Gesellschaft, wie es aktuell der Fall ist. Seit Jahren ist die Zahl der Kirchenmitglieder und Gottesdienstbesucher rückläufig, und es macht sich eine Indifferenz gegenüber Kirche breit (vgl. Pollack 2016, S. 13; EKD 2014, S. 19). Zugleich werden vor allem gegen die römisch-katholische Kirche immer wieder Vorwürfe des sexuellen Missbrauchs erhoben, und der Umgang mit bzw. die Aufarbeitung von entsprechenden Verdachtsfällen durch die Kirche und ihre Repräsentanten kritisiert (vgl. Wernicke und Zoch 2021). Trotz dieser Entwicklungen sind noch immer mehr als die Hälfte der Deutschen Kirchenmitglieder (vgl. EKD 2021, S. 4). Darüber hinaus schätzen sowohl Christen als auch Atheisten die diakonische bzw. karitative Arbeit der Kirche (vgl. EKD 2014, S. 90). Ihre gesellschaftliche Bedeutung als Institution bleibt also (vorerst) bestehen. Mit der dennoch schwer abzustreitenden zunehmenden persönlichen Entfremdung von Kirche spielt „,das Medienbild der Organisation ,Kirche“ und ihrer Vertreter eine immer größere Rolle für die Wahrnehmung der Kirchen und das Verhalten ihrer Mitglieder“ (Kolmer 2014, S. 244). Ein Vorwurf, der dabei immer wieder im Raum steht, ist der einer ,,pauschalen Medienkritik“ an Kirche, ,die ihre 
Reputation mindert und Austritte fördert" (Bösch 2010, S. 452). Um diesem Vorwurf nachzugehen und gleichzeitig eine Forschungslücke zu verkleinern (vgl. Koch 2009, S. 365), haben wir die Kirchenberichterstattung am Beispiel der wichtigsten deutschen Nachrichtensendung Tagesschau zu drei verschiedenen Messzeitpunkten (1998/99, 2008/09 und 2018/19) mit einer quantitativen Inhaltsanalyse untersucht.

Dass über die Kirchen in Deutschland berichtet wird, steht bislang außer Frage: Zum einen stufen Journalisten trotz ihrer eigenen, vermuteten und z.T. auch nachgewiesenen Kirchenferne einen Großteil der kirchlichen Themen prinzipiell als relevant ein (vgl. Roth 2004, S. 339). Zum anderen sichern gesetzliche Regelungen die Stellung der Kirchen im dualen Rundfunksystem (vgl. Link 1995b). So sind ihre Vertreter z. B. fester Teil der Rundfunkgremien (vgl. Link 1995b, S. 299-304), und der Rundfunk ist in Deutschland dazu aufgerufen, ,die sittlichen und religiösen Überzeugungen der Bevölkerung zu achten“ und mithilfe des Angebots „,die Achtung [...] vor Glauben und Meinungen anderer zu stärken“ (Die Medienanstalten 2020, S. 13). Das daraus entstehende Spannungsverhältnis zwischen abnehmender Bedeutung von Kirche und gleichzeitig stark gesicherter Position im Rundfunksystem zeigt, dass es weniger die Frage ist, $o b$, sondern eher wie intensiv und prominent bzw. in welchen Kontexten wie über die beiden Kirchen berichtet wird. Der vorliegende Beitrag widmet daher sich der medialen Darstellung der evangelischen und katholischen Kirche in Deutschland am Beispiel des öffentlich-rechtlichen Informationsflaggschiffs Tagesschau anhand vier zentraler Forschungsfragen, die neben der Intensität der Berichterstattung und der Art und Tonalität der Darstellung auch Veränderungen im zeitlichen Verlauf in den Blick nehmen:

FF1 Wie intensiv und prominent berichtet die Tagesschau über die römisch-katholische und die evangelische Kirche in Deutschland?

FF2 $\mathrm{Zu}$ welchen Anlässen und in welchen Kontexten berichtet die Tagesschau über die römisch-katholische und die evangelische Kirche und wie wird Kirche in diesem Zusammenhang dargestellt?

FF3 Inwieweit unterscheidet sich die Berichterstattung über a) die römisch-katholische und b) die evangelische Kirche?

FF4 Inwieweit sind Veränderungen in der Kirchenberichterstattung der Tagesschau im Zeitverlauf feststellbar?

\section{Religiosität in der deutschen Gesellschaft - Kirche im Wandel}

In Deutschland ist vielfach ein Prozess der Säkularisierung feststellbar (vgl. Pickel 2017, S. 44; Pollack 2016, S. 14). So ist die Zahl der Kirchenmitglieder in den letzten Jahren stetig zurückgegangen (vgl. EKD 2021, S. 4). Im Jahr 2020 waren etwas mehr als 50\% der Deutschen Mitglied einer der beiden Volkskirchen. Wiederum etwas mehr als die Hälfte von ihnen sind Katholiken - rund 22 Mio. -, während 20 Mio. der evangelischen Kirche angehören (vgl. EKD 2021, S. 4; DBK 
2020, S. 73). Gleichzeitig nimmt die Zahl der Konfessionslosen in Deutschland konstant zu (vgl. Pickel 2017). Am häufigsten treten Menschen aus der Kirche aus, weil Religion keine Bedeutung (mehr) für sie hat (,religiöse Indifferenz") und die Kirche „unglaubwürdig“ erscheint (EKD 2014, S. 81). Dennoch ist das Christentum noch immer ,eine gesellschaftsweit akzeptierte Größe“ (Pollack 2016, S. 14). So stehen Konfessionslose der christlichen Verkündigung oder ethischen Äußerungen zwar ambivalent gegenüber, aber sie erwarten überwiegend, dass sich die Kirche für Arme, Kranke und Bedürftige einsetzt (vgl. EKD 2014, S. 90). Kirche wird also trotz religiöser Indifferenz im Rahmen der Diakonie akzeptiert und als feste gesellschaftliche Institution anerkannt.

Obwohl es bislang kaum Forschung zur Religiosität von deutschen Journalisten gibt, dürfte in Analogie zur gesamtdeutschen Entwicklung auch bei ihnen ein Säkularisierungsprozess zu beobachten sein. So stellten Weischenberg et al. (2006) fest, dass deutsche Journalisten vor allem aus der Mittelschicht stammen (S. 69). Schaut man sich die entsprechenden Sinus-Milieus der Mittelschicht an, zeigt sich ein gemischtes Bild ihrer Religiosität - von überwiegender Kirchenferne bis hin zu vereinzelter Kirchennähe (vgl. Hempelmann und Flaig 2019). Untermauert wird die Vermutung, dass auch unter Journalisten die Religiosität abnimmt, durch eine kleine, nicht repräsentative Befragung ostdeutscher Journalisten aus dem Jahr 2004 (besonders zu berücksichtigen ist die soziokulturelle Prägung durch die SED-Diktatur): Fast $80 \%$ der befragten Journalisten gaben hier an, kein Mitglied einer Kirche zu sein, fast $28 \%$ standen nach eigenen Angaben der Kirche kritisch gegenüber (vgl. Roth 2004, S. 332, 334). ${ }^{1}$

Es liegt die Vermutung nahe, dass diese zunehmende Säkularisierung und veränderte Relevanzzuschreibung für die Themensetzung und die Bewertungen in der Kirchenberichterstattung in Deutschland nicht folgenlos geblieben sind. Wie die Berichterstattung aussieht, ist dabei (auch im Hinblick auf den beobachteten Säkularisierungsprozess) durchaus relevant, kann man doch annehmen, dass die Massenmedien für einen großen Teil der Bevölkerung die primäre (vielleicht sogar einzige) Informationsquelle über Kirche sind. Denn je weniger Menschen in Deutschland aktive Kirchenmitglieder sind, je weniger Primärerfahrungen sie also mit der Institution Kirche machen, desto wahrscheinlicher wird es, dass ihre Informationen über Kirche vor allem aus den Medien stammen. Das mediale Bild könnte folglich die Vorstellungen der Deutschen über Kirche maßgeblich prägen.

\section{Die Stellung der Kirche im öffentlich-rechtlichen Rundfunk}

Trotz der sich wandelnden Rolle der Kirche auf Individualebene und in der Gesellschaft existieren langjährig unveränderte gesetzliche Regelungen zum Verhältnis zwischen Kirche und Rundfunk. Vor allem der öffentlich-rechtliche Rundfunk hat die Aufgabe, eine Informations-Grundversorgung zu gewährleisten, um die individuelle und öffentliche Meinungsbildung zu ermöglichen (vgl. Die Medienanstalten

\footnotetext{
1 Ähnliches zeigt eine ältere Studie für Österreich (Gottschlich 1995). Gegenteiliges gilt z. B. für die USA (vgl. Underwood 2002).
} 
2020, S. 7), was die Berichterstattung über Kirche unumgänglich macht. Geregelt wird die Beziehung von Kirche und Rundfunk auf drei Ebenen: (1) auf Ebene der Landesverfassungen und des Grundgesetzes sowie der Staatskirchenverträge, (2) auf Ebene der Rundfunkstaatsverträge (bzw. seit Kurzem der Medienstaatsverträge), der Rundfunk- und Mediengesetze der Länder und der Satzungen der Rundfunkanstalten sowie (3) auf Ebene des europäischen Rechts (vgl. Link 1995b, S. 285).

Auf der ersten Ebene bildet Art. 5 Abs. 1 des Grundgesetzes die wichtigste Grundlage. Dieser Artikel ist in fast allen Landesverfassungen wörtlich oder sinngemäß aufgenommen. Lediglich in Bayern, Brandenburg und Thüringen wurde er dahingehend ergänzt, dass eine Beteiligung der bedeutsamen politischen, weltanschaulichen und gesellschaftlichen Gruppen (darunter auch die Kirchen) in Kontrollgremien des öffentlich-rechtlichen und privaten Rundfunks gegeben und eine ausgewogene Berichterstattung gewährleistet sein muss (vgl. Link 1995b, S. 285-86). Als Hinweis darauf, dass die Kirchen eine gesellschaftlich relevante Gruppe sind, dienen die für den Staat objektiven Kirchenmitgliedszahlen, die nach wie vor darauf hindeuten, dass ,die Kirchen im Konzert der Meinungen ohne Zweifel eine herausragende Stellung“ (Willenberg 2001, S. 153) einnehmen. In den Staatskirchenverträgen wird darüber hinaus das „Drittsenderecht“ der Kirchen in den privaten, aber v.a. öffentlich-rechtlichen Rundfunkanstalten und ihre Vertretung in den Kollegialorganen (Rundfunkrat und Programmausschuss) geregelt (vgl. Link 1995b, S. 287-88). Im Konkordat mit der Freien und Hansestadt Hamburg, wo der NDR die Tagesschau produziert (vgl. NDR 2019), wird außerdem in Artikel 11 versichert, ,dass in den Programmen auf die sittlichen und religiösen Überzeugungen der Bevölkerung Rücksicht genommen wird“ (Vertrag 2006, S. 435).

Schaut man auf die weiteren Ebenen, heißt es beispielsweise im NDR-Staatsvertrag in $\S 8$ Absatz 1:

Er [der NDR] hat sicherzustellen, dass 1. die bedeutsamen politischen, weltanschaulichen und gesellschaftlichen Kräfte und Gruppen aus dem Sendegebiet im Programm angemessen zu Wort kommen können, 2. das Programm nicht einseitig einer Partei oder Gruppe, einer Interessengemeinschaft, einem Bekenntnis oder einer Weltanschauung dient und 3. in seiner Berichterstattung die Auffassungen der wesentlich betroffenen Personen, Gruppen oder Stellen angemessen und fair berücksichtigt werden [...]. (NDR-StV 2005, S. 263)

Dies kann man auch auf die Kirchen als wichtige gesellschaftliche Kraft (vgl. Link 1995a, S. 273) beziehen. Des Weiteren ist in $\S 17$ Absatz 1 geregelt, dass jeweils zwei von maximal 58 Mitgliedern des Rundfunkrats aus der evangelischen Kirche und der römisch-katholischen Kirche stammen müssen (NDR-StV 2005, S. 263). Die Stellung der Kirchen im öffentlich-rechtlichen Rundfunk ist somit trotz der gesellschaftlichen Umbrüche klar gefestigt. Es deutet sich allerdings ein Spannungsverhältnis zwischen Individual- und Organisationsebene an. 


\section{Hintergründe und Wirkungen der Kirchenberichterstattung}

Journalistisches Arbeiten ist geprägt von Entscheidungen, welche Themen, Ereignisse und Akteure überhaupt Eingang in die Berichterstattung finden (Selektionsentscheidung) und wie diese konkret aufbereitet bzw. dargestellt werden (Konstruktionsentscheidung) (vgl. Schäfer 2018, S. 205-208). Das dürfte auch im konkreten Fall der Kirchenberichterstattung nicht anders sein. Bei diesen Entscheidungen können verschiedene Einflussfaktoren auf Mikro-, Meso- und Makroebene von Bedeutung sein, die sich mehr oder weniger stark auf die Berichterstattung auswirken (vgl. z. B. Donsbach 1987, S. 112; Weischenberg 1992, S. 68). Hierzu zählen beispielsweise gesellschaftliche Stimmungen und rechtliche Regelungen, Ereignisund Themeneigenschaften, allgemeine journalistische Relevanzkriterien, die konkreten Produktionsbedingungen und die redaktionellen und wirtschaftlichen Ziele und Zwänge innerhalb der jeweiligen Medienorganisationen, aber auch das Wissen und die Einstellungen der einzelnen Journalisten.

In der Kommunikationswissenschaft existieren verschiedene Ansätze, die zum Ziel haben, die journalistische Nachrichtenentscheidung und ihre Einflussfaktoren in den Blick zu nehmen, wobei je nach Ansatz die Selektionsentscheidung und/ oder die Konstruktionsentscheidung sowie bestimmte Einflussfaktoren und Ebenen im Mittelpunkt stehen. Hierzu zählen z. B. der Gatekeeper-Ansatz (vgl. z. B. Bruns 2009; Robinson 1973; White 1950) und die Nachrichtenwerttheorie (vgl. z. B. Galtung und Ruge 1965; Eilders 1997; Kepplinger 1998; Schulz 1976; Staab 1990), die tendenziell stärker auf die Nachrichtenauswahl abzielen, ebenso wie etwa der NewsBias-Ansatz (vgl. Klein und Maccoby 1954; Rosengren 1979; Schönbach 1977) und der Framing-Ansatz (vgl. z. B. Entman 1993; Scheufele 1999, 2003), die auch die Nachrichtengestaltung stärker in den Blick nehmen (vgl. Schäfer 2018, S. 208).

Die Hintergründe journalistischer Berichterstattung sind jedoch überhaupt nur deshalb von besonderer wissenschaftlicher und gesellschaftlicher Relevanz, weil man davon ausgehen muss, dass die konkreten Medieninhalte, die aus der journalistischen Nachrichtenentscheidung resultieren, wiederum einen Einfluss darauf haben können, wie Menschen bestimmte Themen, Ereignisse und Akteure wahrnehmen und bewerten und wie sie ggf. handeln (vgl. Donsbach 1987; McCombs und Shaw 1972; Scheufele 1999).

Der Framing-Ansatz (vgl. Scheufele 1999, 2003) eignet sich hierbei besonders gut, um diese Zusammenhänge zwischen Nachrichtenentscheidung, Medieninhalten und deren möglichen Wirkungen durch bestimmte Betonungen und Rahmungen im Kontext der Kirchenberichterstattung zu verdeutlichen. Dies begründet im vorliegenden Fall auch, wieso es sinnvoll ist, sich mit den Inhalten der Berichterstattung über Kirche in Deutschland zu beschäftigen. Der Framing-Ansatz geht dabei von einem mehrstufigen Prozess aus, in dessen Verlauf bestimmte Medien-Frames entstehen, die sich vermittelt über die Berichterstattung und deren Wahrnehmung in der Folge auch in Rezipienten-Frames niederschlagen können (Abb. 1). Die Vorstellung ist dabei, dass die Medienberichterstattung nicht nur über ihre Themensetzung Einfluss darauf haben kann, worüber Rezipienten nachdenken (vgl. McCombs und Shaw 1993; McCombs und Reynolds 2009), sondern, vermittelt über Deutungsrahmen in 


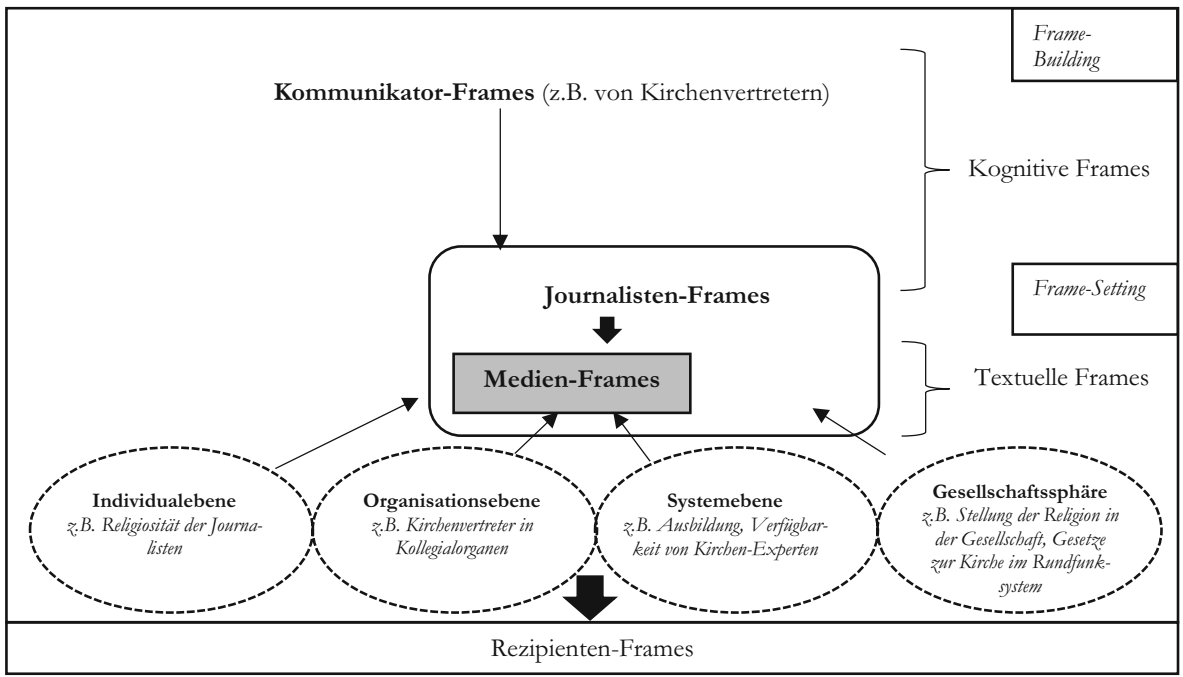

Abb. 1 Framing-Prozess in der Kirchenberichterstattung

der Berichterstattung, auch darauf, wie sie Informationen interpretieren, bestimmte Themen, Ereignisse und Akteure wahrnehmen und bewerten.

Allein schon aufgrund der Komplexität der Realität selektieren Journalisten Informationen und stellen bestimmte Aspekte besonders in den Vordergrund, während andere weniger betont werden (vgl. Matthes 2014, S. 9). Themen werden in der Berichterstattung folglich ,geframt“ (vgl. Entman 1993). Framing hat dabei die Funktion, Informationen zu strukturieren, Komplexität zu reduzieren und Orientierung zu geben (vgl. Dahinden 2006, S. 308). Dies findet sowohl bewusst als auch unbewusst statt (vgl. Kerr 2009, S. 213-214; Kunczik und Zipfel 2005, S. 272). Das Ergebnis dieses Selektions- und Konstruktionsprozesses sind Medien-Frames (im Sinne von Sinnhorizonten oder Deutungsmustern), die eine bestimmte Sicht auf ein Thema nahelegen (vgl. Potthoff 2012, S. 42; Matthes 2007, S. 143, 2014, S. 10).

Framing findet auf allen Stufen des Kommunikationsprozesses statt (vgl. Kerr 2009, S. 213-214; Scheufele 1999, S. 115-117). Auf Seite der Nachrichtenproduktion zu unterscheiden sind dabei kognitive und textuelle Frames, die jedoch in einem gegenseitigen Abhängigkeitsverhältnis stehen (vgl. Potthoff 2012, S. 21; Scheufele 2003, S. 59; Dahinden 2006, S. 308). Alle am Kommunikationsprozess beteiligten Akteure (hier z. B. Kirchen, Journalisten) haben ein bestimmtes Wissen bzw. bestimmte Ein- und Vorstellungen zu einem Thema (kognitive Frames), die sich mehr oder weniger deutlich in den Medieninhalten (textuelle Frames) niederschlagen können (vgl. Matthes 2014, S. 18; Potthoff 2012, S. 54; Scheufele 2003, S. 59). Wenn z. B. die Kirchen ihre Kommunikator-Frames über alle Arten von Öffentlichkeitsarbeit verbreiten, werden diese von den Journalisten in Abhängigkeit ihrer eigenen Vorstellungen und des eigenen Wissens aufgegriffen, modifiziert und ergänzt (vgl. Matthes 2014, S. 14; Scheufele 1999, S. 115). Dabei entstehen sowohl themenspezifische als auch themenunabhängige/generische textuelle Frames, die die Darstellung von Kirche in der Berichterstattung beeinflussen können (vgl. Potthoff 
2012, S. 20; Matthes 2014, S. 59). Ein Mittel, um eine Sichtweise (ein Frame) dabei besonders zu untermauern, sind die „opportunen Zeugen“ (vgl. Hagen 1992). Sie können als Mittel zum Zweck bezeichnet werden, wobei der Zweck das Framing ist. Dies hat schon Entman (1993) erkannt: „The text contains frames, which are manifested by [...] sources of information [...]“ (S. 52)

Neben den kognitiven Frames der Journalisten bestimmen zusätzlich viele weitere Faktoren auf Individual-, Medienorganisations-, Mediensystem- und Gesellschaftsebene, wie die Berichterstattung (über Kirche) am Ende in Form von textuellen Frames konkret aussieht (vgl. z. B. Donsbach 1987, S. 112; Weischenberg 1992, S. 68). So stehen dem geringen Interesse an Kirche auf Individual- und Gesellschaftsebene (in der Bevölkerung und somit aller Wahrscheinlichkeit nach auch bei den Journalisten) wie beschrieben etwa die gesetzlichen Regelungen der Rundfunk- bzw. Medienstaatsverträge entgegen. Auf Medienorganisationsebene machen Kirchenvertreter ihren Einfluss u.a. in Rundfunkräten geltend. Gleichzeitig wird in Bezug auf die Mediensystemebene diskutiert, inwiefern Journalisten durch die zunehmende Entfremdung von Kirche und dem geringen Stellenwert in der Ausbildung über genügend Fachwissen zur sachgerechten Aufarbeitung kirchlicher Themen verfügen (vgl. Dahinden 2009, S. 4; Klenk 2008, S. 52). Zu berücksichtigen sind außerdem Publikumsinteressen und die Orientierung der Organisationen an den Publikumspräferenzen, die in einer Boulevardisierung der Fernsehnachrichten münden kann (vgl. Leidenberger 2015; Landmeier und Daschmann 2011; Esser 1999). Wird beispielsweise im Zuge der Personalisierung (als Teilaspekt der Boulevardisierung) vermehrt über den Papst anstelle der Institution Kirche berichtet, verlagert sich der Schwerpunkt auf Teilaspekte der Kirche - das Kirchenoberhaupt selbst. Die Entstehung der Kirchenberichterstattung als Ganzes ist somit insgesamt ein vielschichtiger Prozess.

Beachtet man nun, dass die Sendezeit der Tagesschau begrenzt ist und Journalisten zwangsläufig Informationen selektieren müssen, dürften in der Berichterstattung bewusst oder unbewusst Informationen und Positionen zu kirchlichen Themen hervorgehoben und somit bestimmte Aspekte, Interpretationen und Bewertungen von Kirche (Medien-Frames) nahegelegt werden. Entsprechend den allgemeinen Annahmen lässt sich vermuten, dass die Entscheidungen, welche Aspekte beim Thema Kirche betont und positiv oder negativ bewertet werden, maßgeblich durch Journalisten-Frames und damit auch die ggf. nachlassende Verbundenheit mit den Kirchen auf Individualebene geprägt sein könnten. Auf der anderen Seite ist davon auszugehen, dass die konkrete Kirchenberichterstattung nicht nur in der Lage ist, das Thema Kirche auf die Agenda der Rezipienten zu heben, sondern mit ihren jeweiligen Akzentuierungen auch maßgeblich darüber mitentscheidet, welche Themen und Aspekte von Kirche die Bevölkerung in Deutschland wahrnimmt und wie sie diese bewertet (Rezipienten-Frames) - was sich wiederum in der gesellschaftlichen Akzeptanz und der Verbundenheit mit der Institution Kirche niederschlagen kann. Entscheidend hierfür ist, wie die konkrete Berichterstattung über Kirche aussieht. 


\section{Forschungsstand: Kirche in den Medien}

Schaut man sich den bisherigen Forschungsstand an, fällt auf, dass zur medialen Darstellung der beiden großen Kirchen bzw. zum Christentum in Deutschland und anderen Ländern fast keine (vor allem kaum aktuelle) Untersuchungen existieren. Außerdem zeichnet sich die bisherige Forschung in ihren Untersuchungsanlagen, den theoretischen Vorüberlegungen und der Operationalisierung durch eine starke Heterogenität aus. Dennoch sollen hier die vier wichtigsten Erkenntnisse aus den vorhandenen Untersuchungen dargestellt werden.

Der erste Punkt betrifft die Quantität der Berichterstattung. Im Jahr 2018 wurden von $93 \mathrm{~h}$ Gesamtsendezeit 69 min $(1,2 \%)$ in der Tagesschau über Religionen und Kirche berichtet. Bei allen anderen Nachrichtensendungen von ARD, ZDF, SAT.1 und RTL liegt der Anteil unter einem Prozent (vgl. Krüger und Zapf-Schramm 2019, S. 52). Insgesamt ist die Berichterstattung über Kirchen in den deutschen Hauptnachrichtensendungen im Laufe der Jahre (2001 bis 2013) gestiegen (vgl. Kolmer 2014, S. 246). Ähnliche Beobachtungen gelten für die Printmedien (vgl. Schielicke 2014, S. 125-126). Inhaltlich fällt dabei auf, dass über die katholische Kirche in den deutschen (und auch Schweizer) Medien häufiger berichtet wird als über die evangelische (vgl. Kolmer 2008, S. 413; Schielicke 2014, S. 128; Koch 2012, S. 26; Favre 2011, S. 78). Dahinden (2009, S. 4) und Favre (2011, S. 78) vermuten, dies liege daran, dass die Protestanten zum einen keinen Papst, keine Skandale und extremen Positionen, zum anderen keine optisch attraktiven Riten vorweisen können. In Verbindung mit den obigen Ausführungen, dass die beiden Kirchen auf Individualebene immer mehr an Bedeutung für Bürger und damit auch Journalisten verlieren (Stichwort, ,religiöse Indifferenz“), zum anderen aber eine gesetzlich geregelte Position im Rundfunksystem haben (Organisationsebene), stellt sich die Frage:

FF1 Wie intensiv und prominent berichtet die Tagesschau über die römisch-katholische und die evangelische Kirche in Deutschland?

Zweitens ist festzuhalten, dass über Religion häufig in Verbindung mit Politik berichtet wird. Diese Schwerpunktsetzung hat im Laufe der Zeit weder zu- noch abgenommen (vgl. Schielicke 2014, S. 129-130). Speziell auf Kirche bezogen kommt hinzu, dass ,,in erster Linie über die Institution, ihre Repräsentanten, Veranstaltungen und Verlautbarungen“ berichtet wird, allerdings kaum ,über Glaubensfragen und christlich ethische Positionen“" (Günther 2000, S. 1; siehe auch Klenk 2006, S. 341). Außerdem liegt der Schwerpunkt der Berichterstattung (zumindest in der Süddeutschen Zeitung) v. a. auf dem kommunikativen Handeln der Kirchen - im Gegensatz zum affektiven, physischen und organisierenden Handeln (vgl. Meier 2006, S. 167). Dabei halten sich innerkirchliche und außerkirchliche Themen in der Berichterstattung die Waage (vgl. Meier 2006, S. 160). Bedenkt man nun, dass die Indifferenz der Bevölkerung und vermutlich auch der Journalisten gegenüber Kirche in den letzten Jahren gestiegen ist, die Journalisten durch die Gesetzgebung aber dennoch zu einer Kirchenberichterstattung angehalten sind, stellt sich die Frage, inwiefern sich dieses Spannungsverhältnis in der Berichterstattung niederschlägt bzw. mit welchem The- 
menkontext Journalisten Kirche framen und so das angedeutete Spannungsverhältnis auflösen. Es stellt sich die Frage:

FF2 $\mathrm{Zu}$ welchen Anlässen und in welchen Kontexten berichtet die Tagesschau über die römisch-katholische und die evangelische Kirche und wie wird Kirche in diesem Zusammenhang dargestellt?

In den bisherigen Studien zeigt sich hierzu drittens ein ambivalentes Bild in Bezug auf Negativität und Konflikthaftigkeit in der Berichterstattung über Kirche und Religion. So kann Schielicke (2014, S. 142) für Tageszeitungen eine signifikante kontinuierliche Zunahme von Konflikten in der Religionsberichterstattung allgemein nachweisen (Ähnliches gilt auch für die Schweiz: vgl. Dahinden 2009, S. 5). Zugleich ist eine über die Jahre konstant überwiegend positive Valenz der Berichterstattung über die katholische wie die evangelische Kirche festzustellen. Zu ähnlich uneindeutigen Ergebnissen kommt Kolmer (2008, 2014), der für die Presse und die Nachrichtensendungen von ARD und ZDF zeigen kann, dass vor allem Personalisierung und Skandalisierung die Berichterstattung über Kirche prägen. Für die deutschen Hauptabendnachrichtensendungen zeichnet sich außerdem ab, dass über die katholische Kirche negativer berichtet wird als über die evangelische (vgl. Kolmer 2008, S. 414, 2014, S. 245; Ähnliches gilt auch für die Schweiz: Koch 2012). Meier (2006) stellt wiederum für die $S Z$ fest, dass die Ereigniswertigkeit bei beiden Konfessionen überwiegend positiv ist, während gleichzeitig die explizite Beurteilung der Kirchen leicht negativ ausfällt (S. 182-183). Untersuchungen aus dem Ausland zeichnen ein ähnliches Bild (vgl. Knott et al. 2013a, b; Kerr 2009; Kerr und Moy 2002; Koch 2012). In Bezug auf die Inhaltsanalyse der Tagesschau stellt sich folglich die Frage, wie kirchliche Handlungen, Aussagen und Akteure dort bewertet und somit geframt werden - besonders vor dem Hintergrund seit Jahren wachsender Skepsis gegenüber der Kirche.

Viertens fällt in der Kirchenberichterstattung eine Tendenz zur Personalisierung auf. Oft steht dabei der Papst im Mittelpunkt, denn ,durch das Papsttum verfügt die Katholische Kirche über eine einfache Möglichkeit, ihr Glaubensangebot in einer personifizierten Weise zu kommunizieren“ (Forschungskonsortium WJT 2007, S. 160). Er wird als Medienberühmtheit und als „Markensymbol des Katholizismus“ inszeniert (Hepp und Krönert 2009, S. 139). In der Schweizer Medienberichterstattung greift knapp jeder dritte Beitrag/Artikel auf das Personalisierungsframe zurück, d.h. ein religiöses Thema wird anhand einer Person erläutert (vgl. Koch 2009, S. 374). Sind ähnliche Tendenzen in der Tagesschau zu beobachten?

Da die Säkularisierungstendenzen seit Jahren zunehmen, stellt sich nicht nur die Frage, ob sich dieser Trend im Laufe der Jahre in der Berichterstattung niedergeschlagen hat. Interessant ist auch zu wissen, inwiefern sich die Berichterstattung über die römisch-katholische und über die evangelische Kirche unterscheidet. In Bezug auf die ersten drei Forschungsfragen soll die Berichterstattung der Tagesschau daher entlang der Konfessionen sowie entlang des Zeitverlaufs betrachtet werden. Es werden folgende Forschungsfragen formuliert: 
FF3 Inwieweit unterscheidet sich die Berichterstattung über a) die römisch-katholische und b) die evangelische Kirche?

FF4 Inwieweit sind Veränderungen in der Kirchenberichterstattung der Tagesschau im Zeitverlauf feststellbar?

\section{Methode}

Antworten auf diese Forschungsfragen sollte im Rahmen einer Vollerhebung für den Zeitraum von insgesamt sechs Jahren zwischen 1998 und 2019 eine quantitative Inhaltsanalyse der 20-Uhr-Ausgabe der Tagesschau geben. Mit durchschnittlich knapp zehn Millionen Zuschauern ist die Tagesschau nach wie vor die Nachrichtensendung mit der größten Reichweite in Deutschland (vgl. Maurer et al. 2020, S. 63) und bietet gleichzeitig für andere Journalisten eine Orientierungsfunktion. Aus forschungsökonomischen Gründen konnte die Berichterstattung allerdings nicht durchgängig untersucht werden, sondern wurde zu drei Messzeitpunkten in einem Abstand von jeweils zehn Jahren analysiert. Jeder Messzeitpunkt umfasste zwei Jahre, sodass die Jahre 1998/99, 2008/09 und 2018/19 (jeweils vollständig) analysiert wurden. Aufgegriffen wurden unter Zuhilfenahme eines eigens entwickelten Stichwortverzeichnisses ${ }^{2}$ alle Beiträge, die explizit über Handlungen oder Äußerungen von Institutionen und/oder Vertretern und Verbänden der römisch-katholischen Kirche weltweit und/oder der evangelischen Kirche in Deutschland oder über kirchliche Festlichkeiten und Feiertage berichteten. Auch Beiträge über eindeutig kirchliche Gebäude wurden aufgegriffen. Dabei musste das Thema Kirche entweder mindestens 50\% des Beitrags ausmachen oder eine der beiden Kirchen direkt oder indirekt zu Wort kommen.

Thematisch decken die untersuchten Jahre auf Seite der Kirchen grundsätzlich eine große Bandbreite ab. Zum ersten Messzeitpunkt war Johannes Paul II. Papst. Er galt als erster „Medien-Papst“ (Bacher et al. 2005, S. 281). Große Ereignisse der Jahre 1998/99 waren u.a. die Schwangeren-Konfliktberatung und diverse Papstreisen. Zum zweiten Messzeitpunkt war der deutsche Benedikt XVI. Papst. Themen waren hier ebenfalls diverse Papstreisen, Katholiken- und evangelische Kirchentage sowie innerkirchliche Konflikte z.B. wegen Missbrauchsfällen und kirchenpolitischer Entscheidungen. Zum dritten und letzten Messzeitpunkt war Papst Franziskus katholisches Kirchenoberhaupt. Im Vordergrund standen in den Jahren 2018/19 diverse Reisen und Treffen des Papstes mit Politikern sowie Kirchenaustrittszahlen, große Feste und Missbrauchsfälle.

Bewusst wurde die Berichterstattung über die römisch-katholische Kirche weltweit, aber über die evangelische Kirche nur in Deutschland untersucht. Diese Entscheidung liegt maßgeblich in den institutionellen Strukturen begründet. Während die katholische Kirche weltweit eine hierarchische Einheit bildet, sodass Entschei-

\footnotetext{
2 Das Stichwortverzeichnis umfasste folgende Stichworte: katholisch, evangelisch, ökumenisch, Papst, Bischof(-skonferenz), Priester, Pfarrer, Kirche(...), Vatikan, Theologe, Gläubige, Gottesdienst, Kardinal, EKD(-Ratsvorsitzender), Caritas, Diakonie, Misereor, Brot für die Welt. Mindestens eines dieser Stichworte musste fallen, damit ein Beitrag aufgegriffen wurde.
} 
dungen im Vatikan auch die katholische Kirche in Deutschland betreffen und nicht von einer eigenständigen deutschen katholischen Kirche gesprochen werden kann, verfügen die Protestanten über keine einheitliche weltweite Struktur.

Wie die meisten Inhaltsanalysen verfolgt auch die vorliegende Untersuchung keine rein deskriptiven Ziele. Wie u. a. Maurer und Reinemann (2006, S. 12) konstatieren, ist nahezu allen Inhaltsanalysen zumindest implizit die Annahme gemein, dass „massenmediale Inhalte entweder die Prozesse und Rahmenbedingungen reflektieren, aus denen diese Inhalte resultieren, oder dass sie Voraussetzungen für Effekte bei den Rezipienten sind“. Tatsächlich liegt der Analyse in diesem Fall sowohl ein diagnostischer als auch ein prognostischer Ansatz zugrunde (vgl. dazu ausführlicher Schäfer 2018, S. 282-283), die sich aus den theoretischen Vorüberlegungen zum Framing-Ansatz und damit den Entstehungsbedingungen und möglichen Wirkungen der Darstellungen von Kirche in Deutschland ergeben. Diagnostisch ist die Perspektive insofern, als die Kirchenberichterstattung der Tagesschau gewisse Rückschlüsse auf die journalistische Nachrichtenentscheidung und deren Einflussfaktoren ermöglichen könnte, deren Resultat sie ist. Hierzu gehört beispielsweise die Frage, inwieweit sich die über die Zeit beobachtbaren Säkularisierungstendenzen auf Gesellschaftsebene, die auch vor Journalisten nicht Halt machen, in der Darstellung niederschlagen. Gleichzeitig verfolgt die Inhaltsanalyse insofern einen prognostischen Ansatz, als Ergebnisse zu Ausmaß, Form und Inhalten der Berichterstattung immer auch bestimmte Wirkungsvermutungen auf Rezipientenseite nahelegen, da entsprechend den theoretischen Vorüberlegungen u.a. davon auszugehen ist, dass sich Medienframes in Rezipientenframes niederschlagen und so das Bild von Kirche in Deutschland prägen können.

Bei der Codierung kamen drei geschulte Codiererinnen zum Einsatz. Codiert wurde auf Beitragsebene. Formale Kategorien waren (1) das Sendungsdatum des Beitrags (Holstis $r_{h}=1,0$; Krippendorfs $\alpha=1,0$ ), (2) die Position des Beitrags in der Sendung $\left(r_{h}=1,0 ; k-\alpha=1,0\right)$, (3) die Länge des Beitrags in Sekunden $\left(r_{h}=0,80\right.$; $\mathrm{k}-\alpha=0,71)$ und (4) die Art des Beitrags (z. B. Meldung/Nachricht oder Bericht) in Anlehnung an Leidenberger (2015) $\left(\mathrm{r}_{\mathrm{h}}=1,0 ; \mathrm{k}-\alpha=1,0\right)$.

Inhaltliche Kategorien bildeten u.a. (5) die Konfession, über die schwerpunktmäßig berichtet wurde $\left(r_{h}=1,0 ; k-\alpha=1,0\right)$, sowie in Anlehnung an Schielicke (2014) und Kerr (2009) die zwei wichtigsten Themen als (6) Haupt- und (7) Nebenthema, erfasst mithilfe eines aus 15 Ausprägungen bestehenden nominalen Schlüsselplans $\left(\mathrm{r}_{\mathrm{h}}=0,93\right.$ bzw. 0,87; k- $\alpha=0,91$ bzw. 0,80). Codiert wurde zudem, (8) inwiefern die Tageschau über kircheninterne und kirchenspezifische Themen berichtet oder Kirche unabhängig von kirchenspezifischen Anlässen Teil der Berichterstattung ist $\left(r_{h}=0,93 ; k-\alpha=0,86\right)$. Des Weiteren lag das Augenmerk (9) in Anlehnung an Kepplinger (1989) auf dem Anlass der Berichterstattung $\left(\mathrm{r}_{\mathrm{h}}=0,90 ; \mathrm{k}-\alpha=0,79\right)$. Dabei war zwischen genuinen, mediatisierten und inszenierten Ereignissen zu unterscheiden. Während genuine Ereignisse unbeeinflusst von der Medienberichterstattung stattfinden, werden mediatisierte Ereignisse für Medien und Öffentlichkeit aufbereitet und inszenierte Ereignisse sogar eigens für diese veranstaltet (vgl. Kepplinger 1989, S. 13). In Anlehnung an Leidenberger (2015) wurde des Weiteren auf einer fünfstufigen bipolaren Skala (10) der Grad der Personalisierung erfasst und im Anschluss zu drei Stufen (,,überwiegend/ausschließlich Sachorientierung“, ,,ausge- 
glichenes Verhältnis zwischen Sachorientierung und Personalisierung“ und „überwiegend/ausschließlich Personalisierung") zusammengefasst $\left(r_{h}=0,93 ; k-\alpha=0,65\right)$, um eine erste Tendenz für Personalisierungsframes (vgl. Koch 2009, S. 373) feststellen zu können. Ein nominaler Schlüsselplan in Anlehnung an Schielicke (2014) half zu ermitteln, ob (11) es eher um Handlungen bzw. Ereignisse oder um Stellungnahmen von Kirchenvertretern geht $\left(r_{h}=0,87 ; k-\alpha=0,69\right)$. Außerdem wurden (12) die Handlungsträger der Berichterstattung mithilfe einer dichotomen Abfrage der Präsenz ranghoher Kirchenvertreter erfasst $\left(r_{h}=0,98 ; k-\alpha=0,95\right)$.

Die (13) Tendenz des gesamten Beitrags in Bezug auf Handlungen und Äußerungen von Kirche ließ sich nach Klenk (2008) auf einer fünfstufigen bipolaren Skala von ,ausschließlich positiv“ bis „ausschließlich negativ“ festhalten und für die Auswertung anschließend in drei Stufen (,ausschließlich/überwiegend positiv“, ,ambivalent", ,ausschließlich/überwiegend negativ“) zusammenfassen $\left(\mathrm{r}_{\mathrm{h}}=0,87\right.$; $\mathrm{k}-\alpha=0,76$ ). Darüber hinaus untersuchten die Codiererinnen (14) mithilfe von drei einzelnen dichotomen Items die Konflikthaftigkeit eines Beitrags zur Erfassung von Konflikt-Framing nach Semetko und Valkenburg (2000, S. 99-100). So wurde nach Uneinigkeiten, verschiedenen Sichtweisen und Vorwürfen innerhalb eines Beitrags gefragt $\left(r_{h}=0,89 ; k-\alpha=0,72\right)$. Schließlich waren (15) die positiven, negativen und neutralen Aussagen von im Beitrag auftretenden Akteuren (Quellen/Zeugen) über Kirche offen zu zählen und zu erfassen $\left(r_{h}=0,98 ; k-\alpha=0,92\right)$. Auf diese Weise ließ sich die Verwendung möglicher opportuner Zeugen messen, die Mittel zum Zweck für bewusstes Framing sein können (vgl. Entman 1993, S. 52).

\section{Ergebnisse}

\subsection{Intensität der Berichterstattung und Platzierung der Beiträge}

Insgesamt stützt sich die Analyse auf 514 Beiträge, die sich fast zu gleichen Teilen auf die drei Messzeitpunkte verteilen (1998/99: 179 Beiträge, 2008/09: 171 Beiträge und 2018/19: 164 Beiträge). Von diesen Beiträgen wurden fast 80\% an vierter oder noch späterer Stelle der Sendung gesendet. Die Platzierung der Kirchen-Beiträge hat sich dabei im Laufe der Jahre innerhalb der Sendung nicht signifikant verändert: So sind z.B. zu jedem Messzeitpunkt lediglich etwa 5 bis $10 \%$ der Beiträge ,Aufmachernachrichten' $\left(\chi^{2}(6)=5,94\right.$; n. s.). Nicht anders sieht es bei der Länge der Beiträge aus: Auch diese bleibt mit im Mittel 65 s (1998/99) bis 74 s (2018/19) relativ konstant $(F(2,511)=2,61 ;$ n. s.). Forschungsfrage 1 kann man also dahingehend beantworten, dass sich die Intensität der Berichterstattung und die Prominenz des Themas innerhalb der Sendung und damit die Relevanz der Kirche innerhalb der Berichterstattung der Tagesschau in den letzten 20 Jahren gemessen an Anzahl, Länge und Platzierung der Beiträge trotz der Säkularisierungstendenzen in der Gesellschaft nicht entscheidend verändert hat.

Vergleicht man die Relevanz der katholischen und der evangelischen Kirche, zeigen sich klare Unterschiede. Obwohl in Deutschland fast genauso viele Katholiken wie Protestanten leben, ist die katholische Kirche mit fast $68 \%$ aller Beiträge, in denen sie im Mittelpunkt steht, in der Berichterstattung deutlich überrepräsentiert, 
während über die evangelische Kirche in nur rund $13 \%$ der Fälle berichtet wird. Acht Prozent der Beiträge behandeln beide Konfessionen zu gleichen Teilen, und rund vier Prozent thematisieren die Ökumene. In den restlichen fast neun Prozent ist die Konfession nicht eindeutig bestimmbar. Dieses Verhältnis zwischen den beiden Konfessionen hat sich im Laufe der Jahre nicht verändert $\left(\chi^{2}(2)=0,15\right.$; n. s. $)$. Einschränkend muss allerdings gesagt werden, dass die katholische Kirche im Gegensatz zur evangelischen Kirche als weltweite Institution untersucht wurde, auch wenn dieser Faktor vermutlich nicht die gesamte Überrepräsentation erklären kann. Hinsichtlich der durchschnittlichen Platzierung der Beiträge in der Sendung und der durchschnittlichen Länge unterscheidet sich die Berichterstattung über die beiden Konfessionen nicht.

\subsection{Anlässe und Themenkontexte der Kirchenberichterstattung}

Anlass der Berichterstattung über Kirche ist meist ein konkretes Ereignis (z. B. der Kirchentag). Beiträge, in denen Stellungnahmen der Kirchen zur Berichterstattung führen (z. B. die Stellungnahme eines deutschen Bischofs zur Frage der Abtreibung) sind vergleichsweise selten, wobei gewisse zeitliche Schwankungen zu beobachten sind (Tab. 1). Die Verteilung zwischen genuinen, mediatisierten und inszenierten Ereignissen als Aufhänger der Berichterstattung hat sich dagegen im Laufe der Jahre nicht signifikant verändert $\left(\chi^{2}(4)=5,78\right.$; n. s.). So berichtete die Tagesschau 1998/99 in $41 \%$ der Fälle über genuine Ereignisse, in $38 \%$ über mediatisierte und in $22 \%$ über inszenierte Ereignisse. 2008/09 entfielen $40 \%$ der Beiträge auf genuine, $45 \%$ auf mediatisierte und $16 \%$ auf inszenierte Ereignisse. Und zum dritten Messzeitpunkt wurde in $33 \%$ der Fälle über genuine Ereignisse, in $48 \%$ über mediatisierte und in $20 \%$ über inszenierte Ereignisse berichtet.

Die Berichterstattung über die Konfessionen unterscheidet sich hinsichtlich des Anlasses der Berichterstattung nicht $\left(\chi^{2}(1)=0,04\right.$; n. s. $)$.

Kircheninterne Themen (z. B. Wahlen für Kirchenämter) sind mit rund $86 \%$ der Fälle im Gegensatz zu kirchenexternen Themen (z. B. Diskussionen über die hohe Arbeitslosenquote in Deutschland) mit knapp 14\% deutlich häufiger Gegenstand der Kirchenberichterstattung. Dies hat sich auch im Laufe der Jahre nicht verändert $\left(\chi^{2}(2)=4,25 ;\right.$ n. s. $)$.

Bei der genaueren Betrachtung der Themenverteilung (Tab. 2) fällt auf, dass kirchliche Veranstaltungen und Feiertage mit mehr als der Hälfte der Fälle als Hauptthemen dominieren. Es folgen mit $13 \%$ Beiträge in Verbindung mit politischen Themen und mit $10 \%$ die Berichterstattung über Kirchenpolitik und Kirchenämter.

Tab. 1 Berichterstattungsanlass in der Kirchenberichterstattung der Tagesschau im Zeitverlauf

\begin{tabular}{|c|c|c|c|c|}
\hline & Jahre & & & \\
\hline Berichterstattungsanlass & $\begin{array}{l}1998 / 99 \\
(n=178) \\
\text { in } \%\end{array}$ & $\begin{array}{l}2008 / 09 \\
(n=170) \\
\text { in } \%\end{array}$ & $\begin{array}{l}2018 / 19 \\
(n=156) \\
\text { in } \%\end{array}$ & $\begin{array}{l}\text { Gesamt } \\
(n=504) \\
\text { in } \%\end{array}$ \\
\hline Ereignis & 70 & 91 & 79 & 80 \\
\hline Stellungnahme & 30 & 9 & 21 & 20 \\
\hline
\end{tabular}

Kreuztabellenstatistik: $\chi^{2}(2)=23,49 ; p<0,001 ; \varphi=0,22$ 
Tab. 2 Themenstruktur (Hauptthema) der Kirchenberichterstattung in der Tagesschau in den Jahren 1998/99, 2008/09 und 2018/19 $(n=514)$

\begin{tabular}{lll}
\hline & $N$ & Prozent \\
\hline Kirchliche Veranstaltungen und Feiertage & 279 & 54 \\
Politik: Gesetze, Regierung, Staatsbesuche & 68 & 13 \\
Kirchenpolitik, Kirchenämter & 51 & 10 \\
Ethik, Gesellschaft und Soziales & 30 & 6 \\
Juristische Handlungen: Kriminalität, Gericht & 21 & 4 \\
Sonstiges & 21 & 4 \\
Krieg, Gewalt, Terror & 18 & 4 \\
Kunst, Kultur und Medien & 8 & 2 \\
Historie & 7 & 1 \\
Katastrophen, schwere humane Krisen & 6 & 1 \\
Wirtschaft, Business, Finanzen & 4 & 1 \\
Wissenschaft & 1 & 0 \\
\hline
\end{tabular}

Bei den Nebenthemen dominieren mit knapp $19 \%$ die Berichterstattung über „Ethik, Gesellschaft und Soziales“ (z. B. Berichte über Beratungsstellen bei Abtreibungen) und mit rund $9 \%$ das Thema „Krieg, Gewalt, Terror“. Fast die Hälfte der Beiträge (46\%) behandelt kein zweites Thema. Rund Dreiviertel der Beiträge beschäftigen sich mit den drei wichtigsten Hauptthemen (kirchliche Veranstaltungen und Feiertage, Politik sowie Kirchenpolitik und Kirchenämter) und den zwei wichtigsten Nebenthemen (Ethik, Gesellschaft und Soziales sowie Krieg, Gewalt und Terror) bzw. keinem Nebenthema, wobei sich die Verteilung der Haupt- $\left(\chi^{2}(4)=8,30\right.$; n. s.) sowie der Nebenthemen $\left(\chi^{2}(4)=9,29\right.$; n. s.) im Laufe der Jahre nicht wesentlich verändert hat. Allerdings zeigen sich konfessionelle Unterschiede: So wird über die katholische Kirche häufiger im Zusammenhang mit kirchenspezifischen Themen berichtet (92\%), als dies bei der evangelischen Kirche (75\%) der Fall ist (Tab. 3).

Das Verhältnis zwischen Personalisierung und Sachorientierung ist über die Zeit in der gesamten Kirchenberichterstattung konstant relativ ausgeglichen $\left(\chi^{2}(4)=2,39\right.$; n.s.). So waren zum ersten Messzeitpunkt beispielsweise $43 \%$ der Beiträge überwiegend oder ausschließlich sachorientiert und $45 \%$ überwiegend oder ausschließlich personalisiert. Zum zweiten Messzeitpunkt wiesen $36 \%$ überwiegend oder ausschließlich Sachorientierung und $50 \%$ überwiegend oder ausschließlich Personalisierung auf. Und 2018/19 konnte bei $42 \%$ der Beiträge eine überwiegende/

Tab. 3 Zusammenhang von Konfession und Themenwahl in der Kirchenberichterstattung der Tagesschau

\begin{tabular}{llll}
\hline Themenwahl & Konfession & & Summe \\
& Katholische & Kirche & Evangelische \\
$(n=351)$ & Kirche & $(n=64)$ & in $\%$ \\
& in $\%$ & in $\%$ & 89 \\
\hline Kircheninternes bzw. -spezifisches Thema & 92 & 75 & 11 \\
Von Kirche unabhängiges Thema & 8 & 25 & \\
\hline
\end{tabular}

Kreuztabellenstatistik: $\chi^{2}(1)=15,43 ; p<0,001 ; \varphi=0,19$ 
Tab. 4 Der Papst als Akteur in der Kirchenberichterstattung der Tagesschau im Laufe der Zeit

\begin{tabular}{|c|c|c|c|c|}
\hline \multirow[b]{2}{*}{ Der Papst als Akteur } & \multicolumn{4}{|l|}{ Jahre } \\
\hline & $\begin{array}{l}1998 / 99 \\
(n=179) \\
\text { in } \%\end{array}$ & $\begin{array}{l}2008 / 09 \\
(n=171) \\
\text { in } \%\end{array}$ & $\begin{array}{l}2018 / 19 \\
(n=164) \\
\text { in } \%\end{array}$ & $\begin{array}{l}\text { Gesamt } \\
(n=514) \\
\text { in } \%\end{array}$ \\
\hline Kommt nicht im Beitrag vor & 58 & 53 & 67 & 59 \\
\hline Kommt im Beitrag vor & 42 & 47 & 33 & 41 \\
\hline
\end{tabular}

Kreuztabellenstatistik: $\chi^{2}(2)=6,84 ; p<0,05 ; \varphi=0,12$

ausschließliche Sachorientierung codiert werden, während $48 \%$ überwiegend oder ausschließlich personalisiert waren.

Allerdings unterscheiden sich die Konfessionen im Grad der Personalisierung signifikant voneinander $\left(n=412 ; \chi^{2}(2)=9,61 ; p<0,01\right.$; Cramer-V=0,15). Während ca. $38 \%$ der Beiträge über die evangelische Kirche überwiegend oder ausschließlich Personalisierung und $41 \%$ überwiegend oder ausschließlich Sachorientierung aufweisen, sind fast $57 \%$ der Beiträge über die katholische Kirche überwiegend/ ausschließlich personalisiert und $32 \%$ überwiegend/ausschließlich sachorientiert. Dies könnte auf einen Papst-Bias zurückzuführen sein und passt zu der Erkenntnis, dass in $45 \%$ aller codierten Beiträge der Papst als Akteur zu finden ist, wobei er im Laufe der Jahre signifikant seltener Teil der Berichterstattung wird (Tab. 4).

\subsection{Die wertende Darstellung kirchlicher Handlungen und Äußerungen}

In der gesamten Kirchenberichterstattung ist einerseits eine zunehmend negative Tendenz im Laufe der Jahre zu beobachten (Abb. 2; Tab. 5) und andererseits feststellbar, dass die Konflikthaftigkeit der Berichterstattung abgenommen hat (Tab. 5). War die Tendenz z. B. zum ersten Messzeitpunkt im Schnitt mit 0,1 $(S D=0,7)$ leicht po-

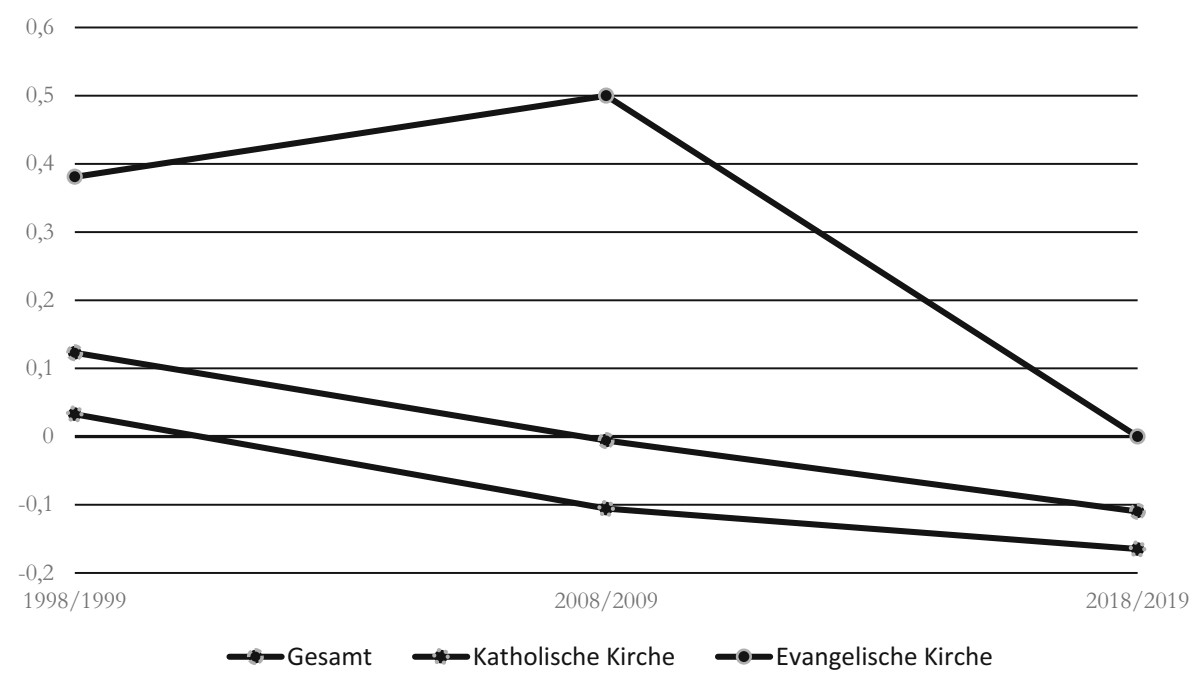

Abb. 2 Tendenz der Kirchenberichterstattung im Zeitverlauf (3-stufige Skala: ,überwiegend/ausschließlich negativ“ (-1), ,ambivalent“ (0), ,überwiegend/ausschließlich positiv“ (1)) 
Tab. 5 Die wertende Darstellung der katholischen und evangelischen Kirche in der Berichterstattung der Tagesschau im Laufe der Jahre

\begin{tabular}{|c|c|c|c|c|}
\hline & $\begin{array}{l}1998 / 99 \\
(n=179)\end{array}$ & $\begin{array}{l}2008 / 09 \\
(n=170-171)\end{array}$ & $\begin{array}{l}2018 / 19 \\
(n=163-164)\end{array}$ & \\
\hline & $\mathrm{M}(\mathrm{SD})$ & $\mathrm{M}(\mathrm{SD})$ & $\mathrm{M}(\mathrm{SD})$ & $\mathrm{F}$ \\
\hline $\begin{array}{l}\text { Tendenz bzw. Tonalität des Beitrags } \\
\text { (gesamt })^{1,4}\end{array}$ & $0,1(0,7)^{\mathrm{a}}$ & $0,0(0,7)$ & $-0,1(0,7)^{\mathrm{b}}$ & $4,62^{* *}$ \\
\hline Evangelische Kirche $e^{1,5}$ & $0,4(0,6)$ & $0,5(0,5)^{\mathrm{a}}$ & $0,0(0,7)^{\mathrm{b}}$ & $4,07^{*}$ \\
\hline Katholische Kirche ${ }^{1}$ & $0,0(0,8)$ & $-0,1(0,7)$ & $-0,2(0,8)$ & 2,09 \\
\hline $\begin{array}{l}\text { Summenindex Konflikthaftigkeit des } \\
\text { Beitrags (gesamt) })^{2,4}\end{array}$ & $1,3(1,2)^{\mathrm{a}}$ & $0,9(1,2)^{\mathrm{b}}$ & $1,0(1,2)$ & $5,49^{* *}$ \\
\hline Evangelische Kirche ${ }^{2,5}$ & $1,2(1,1)^{\mathrm{a}}$ & $0,4(0,9)^{\mathrm{b}}$ & $1,2(1,4)$ & $3,59^{*}$ \\
\hline Katholische Kirche ${ }^{2}$ & $1,4(1,2)$ & $1,0(1,3)$ & $1,3(1,2)$ & 2,91 \\
\hline $\begin{array}{l}\text { Negative Aussagen über Kirche in } \\
\text { einem Beitrag (gesamt) }\end{array}$ & $0,3(1,0)$ & $0,3(0,9)$ & $0,5(1,2)$ & 1,00 \\
\hline Evangelische Kirche ${ }^{3}$ & $0,1(0,2)$ & $0,1(0,6)$ & $0,5(1,1)$ & 1,93 \\
\hline Katholische Kirche ${ }^{3}$ & $0,5(1,2)$ & $0,4(1,0)$ & $0,5(1,2)$ & 0,13 \\
\hline $\begin{array}{l}\text { Positive Aussagen über Kirche in } \\
\text { einem Beitrag (gesamt) }{ }^{3}\end{array}$ & $0,2(0,6)$ & $0,3(0,7)$ & $0,2(0,6)$ & 1,10 \\
\hline Evangelische Kirche ${ }^{3}$ & $0,3(0,7)$ & $0,4(0,9)$ & $0,1(0,3)$ & 0,98 \\
\hline Katholische Kirche ${ }^{3}$ & $0,2(0,6)$ & $0,3(0,6)$ & $0,2(0,5)$ & 0,76 \\
\hline
\end{tabular}

Gruppen mit unterschiedlichen Kennbuchstaben $(a, b)$ innerhalb einer Zeile unterscheiden sich signifikant auf dem $5 \%$-Niveau

$n=513-514, n_{\text {kath }}=347-348, n_{\mathrm{ev}}=64$; einfaktorielle Varianzanalyse (Post-Hoc-Test: Bonferroni/Tamhane); ${ }^{* *} p<0,01, * p<0,05 ;{ }^{4} \eta^{2}=0,02 ;{ }^{5} \eta^{2}=0,11-0,12$

${ }^{1}$ Der Gesamteindruck des Beitrags in Bezug auf Handlungen und Äußerungen von Kirche (,,überwiegend/ ausschließlich negativ“ (-1), ,ambivalent“ (0), ,überwiegend/ausschließlich positiv“ (1))

${ }^{2}$ Der Summenindex Konflikthaftigkeit besteht aus den drei Items ,Zeigt der Beitrag Uneinigkeit ...“, „Enthält der Beitrag zwei oder mehr Sichtweisen auf ein Thema oder Problem?“ und „Wirft einer ... einem andern ... etwas vor?"

${ }^{3}$ Die Aussagen von Quellen/Zeugen wurden gezählt und offen abgefragt

sitiv, ist sie 2018/19 mit $-0,1(S D=0,7)$ leicht negativ. Gleichzeitig werden im Laufe der Jahre weniger Konflikte dargestellt. Liegt der Konflikt-Summenindex 1998/99 im Schnitt noch bei 1,3 (SD=1,2), fällt er 2008/09 auf 0,9 $(S D=1,2)$ und steigt 2018/19 lediglich leicht wieder auf 1,0 $(S D=1,2)$. Betrachtet man die beiden Konfessionen getrennt in Bezug auf die Tendenz im Zeitverlauf, zeigt sich, dass Beiträge über die evangelische Kirche im Laufe der Jahre signifikant negativer werden. Auch in Bezug auf die katholische Kirche ist eine Tendenz zu einer negativeren Berichterstattung feststellbar, wenngleich die beobachtbaren Unterschiede in diesem Fall nicht signifikant sind (Tab. 5).

Auffällig ist, dass in der Kirchenberichterstattung der Tagesschau insgesamt signifikant mehr Menschen zitiert werden, die sich negativ über die Kirchen äußern, als solche, die Positives aussagen $(t(512)=2,80 ; p<0,01)$. So werden im Schnitt 0,2 $(S D=0,6)$ positive Meinungen über die Kirchen pro Beitrag, aber 0,4 $(S D=1,0)$ negative Meinungen zitiert. Dies hat sich im Laufe der Jahre nicht geändert (Tab. 5).

Beim Vergleich der beiden Konfessionen fällt auf, dass die Tendenz in Beiträgen über die katholische Kirche insgesamt signifikant negativer ist als in solchen über 
Tab. 6 Die wertende Darstellung der katholischen und evangelischen Kirche in der Berichterstattung der Tagesschau im Vergleich

\begin{tabular}{llllll}
\hline & \multicolumn{4}{l}{ Katholische Kirche } & \multicolumn{3}{l}{ Evangelische Kirche } & \\
& M & SD & M & SD & t-Test \\
\hline $\begin{array}{l}\text { Tendenz bzw. Tonalität des } \\
\text { Beitrags }\end{array}$ & $-0,1$ & 0,8 & 0,3 & 0,6 & $3,87^{* * * *}$ \\
$\begin{array}{l}\text { Summenindex Konflikthaftigkeit } \\
\text { des Beitrags }\end{array}$ & 1,2 & 1,3 & 0,9 & 1,2 & $2,00^{*}$ \\
$\begin{array}{l}\text { Negative Aussagen über Kirche in } \\
\text { einem Beitrag }\end{array}$ & 0,5 & 1,1 & 0,2 & 0,7 & $2,38^{*}$ \\
$\begin{array}{l}\text { Positive Aussagen über Kirche in } \\
\text { einem Beitrag }\end{array}$ & 0,2 & 0,6 & 0,3 & 0,7 & 0,48 \\
\hline
\end{tabular}

$n_{\text {kath }}=347-348, n_{\text {ev }}=64 ; * p<0,05, * * * p<0,001$

${ }^{1}$ Der Gesamteindruck des Beitrags in Bezug auf Handlungen und Äußerungen von Kirche (,,überwiegend/ ausschließlich negativ“ (-1), ,ambivalent“ (0), ,überwiegend/ausschließlich positiv“ (1))

${ }^{2}$ Der Summenindex Konflikthaftigkeit besteht aus den drei Items ,Zeigt der Beitrag Uneinigkeit ...“, ,Enthält der Beitrag zwei oder mehr Sichtweisen auf ein Thema oder Problem?“ und „Wirft einer ... einem andern ... etwas vor?"“

${ }^{3}$ Die Aussagen von Quellen/Zeugen wurden gezählt und offen abgefragt

die evangelische Kirche (Tab. 6), obwohl - wie bereits beschrieben - besonders die Tendenz der Beiträge über die evangelische Kirche im Laufe der Jahre signifikant negativer geworden ist. Die insgesamt negativere Berichterstattung über die katholische Kirche passt zu der Erkenntnis, dass Beiträge über die katholische Kirche mit einem Mittelwert von 1,2 $(S D=1,3)$ signifikant konflikthaltiger sind als Beiträge über die evangelische Kirche $(M=0,9 ; S D=1,2)$ und dass Beiträge über die katholische Kirche signifikant mehr negative Aussagen enthalten $(M=0,5 ; S D=1,1)$ als Beiträge über die evangelische Kirche $(M=0,2 ; S D=0,7)$. Keine signifikanten Unterschiede zwischen den Konfessionen zeigen sich jedoch in der Zitation positiver Meinungen in einem Beitrag (Tab. 6).

Es zeichnet sich zusammenfassend ab, dass die Bewertung der Kirchen in den Beiträgen der Tagesschau insgesamt im Laufe der Jahre negativer geworden ist.

\section{Fazit}

Die Ergebnisse der Inhaltsanalyse zeigen, dass sich die Relevanz der Kirche innerhalb der Berichterstattung der Tagesschau in den drei Untersuchungszeiträumen gemessen an Anzahl, Länge und Platzierung der Beiträge trotz der beobachteten Säkularisierungstendenzen in der Gesellschaft nicht entscheidend verändert hat. Auch wenn die religiöse Indifferenz in Deutschland in den letzten Jahren zugenommen hat, bleibt die Bedeutung der Kirchen als gesellschaftlich relevante Institutionen in der Berichterstattung bestehen (FF1). Dies könnte womöglich eine Folge der gesetzlich geregelten Stellung der Kirchen im dualen Rundfunksystem und den Rundfunkräten der Institutionen sein. Ob dies so ist oder ob Journalisten den Kirchen als Individuen und im Redaktionsalltag gleichbleibend Relevanz zuschreiben, müsste gesondert zum Beispiel in einer Befragungsstudie untersucht werden. 
Die katholische Kirche ist im Vergleich zur evangelischen Kirche in der Berichterstattung überrepräsentiert, was mit den bisherigen Forschungsergebnissen im Einklang steht (vgl. Schielicke 2014), der realen gesellschaftlichen Verteilung von Kirchenmitgliedern in Deutschland aber widerspricht (FF3). Dies könnte zum einen daran liegen, dass Berichte über die katholische Kirche in ihrer weltweiten Struktur, Berichte über die evangelische Kirche aber nur innerhalb Deutschlands untersucht wurden. Zum anderen kann die Überrepräsentation der katholischen Kirche aber auch durch die häufige Berichterstattung über den Papst und die kontroversen Themen der katholischen Kirche erklärt werden, die klassische journalistische Selektionsmechanismen bedienen (vgl. Galtung und Ruge 1965; Kepplinger 1998).

Über beide Kirchen wird oft nur in Verbindung mit kircheninternen Themen berichtet, was sich auch im Laufe der Jahre nicht geändert hat. Diese Erkenntnisse scheinen nicht in die gesellschaftlichen Entwicklungen zu passen: Während immer mehr Menschen den Kirchen distanziert gegenüberstehen, sind es gerade die kircheninternen Themen und Ereignisse, die es in die Berichterstattung schaffen (FF2 und FF4). Vermuten lässt sich an dieser Stelle nur, dass diese Beobachtung mit den gesetzlichen Regelungen zusammenhängt, sodass an wichtigen Feiertagen und bei großen Veranstaltungen gewissermaßen automatisiert über die Kirchen berichtet wird, obwohl dies weniger zu den gesellschaftlichen Erwartungen an Kirche passt.

Thematisch wird das Bild der Kirchen in der Tagesschau (in Übereinstimmung mit der bisherigen Forschung) so geframt, dass am meisten über kirchliche Veranstaltungen, politische Diskussionen und Kirchenpolitik berichtet wird. Das Bild der großen Institutionen wird somit verstärkt und gleichzeitig auf interne Themen und Konflikte reduziert. Allerdings scheinen die Journalisten zwischen den zwei Konfessionen zu unterscheiden, da die evangelische Kirche signifikant häufiger mit kirchenunabhängigen Themen in Verbindung gebracht wird als die katholische Kirche. Entgegen der sonstigen allgemeinen Forschungserkenntnisse zur Boulevardisierung lassen sich keine Personalisierungstendenzen der Kirchenberichterstattung nachweisen (FF2).

Die Berichterstattung über Kirche ist eher negativ und kritisch, wobei die katholische Kirche signifikant negativer dargestellt wird als die evangelische, was im Einklang mit den Ergebnissen von Kolmer (2008, 2014) steht. Die Tendenz der gesamten Kirchenberichterstattung ist im Laufe der Jahre signifikant negativer geworden. Zu beobachten ist hier zudem, dass in der Berichterstattung häufiger negative Äußerungen über die Kirchen zitiert werden. Diese Ergebnisse scheinen grundsätzlich zu den Säkularisierungstendenzen zu passen und deuten darauf hin, dass sich mögliche Journalisten-Frames auch in den textuellen Frames der Kirchen niederschlagen könnten (FF2, FF3 und FF4). Ob die zunehmende Negativität allerdings mit einer persönlichen kritischeren Sichtweise der Journalisten, etwaigen Veränderungen auf Redaktionsebene und/oder schlichtweg einer zunehmenden negativen Ereignislage (z.B. durch die vermehrten Vorwürfe sexuellen Missbrauchs) zu erklären ist, kann im Rahmen dieser Inhaltsanalyse freilich nicht abschließend beantwortet werden. Ergänzende quantitative und/oder qualitative Journalistenbefragungen könnten hierüber in Zukunft Aufschluss geben und die journalistische Nachrichtenentscheidung und deren Einflussfaktoren expliziter in den Blick rücken.

Mit Blick auf die möglichen Wirkungen der medialen Darstellungen von Kirche in Deutschland lässt sich im Hinblick auf die Darstellung der Tagesschau vor dem 
Hintergrund der theoretischen Überlegungen vermuten, dass Kirche zwar durch das regelmäßige (ggf. routinierte) Aufgreifen anlässlich bestimmter Ereignisse bei den Rezipienten nach wie vor präsent sein dürfte. Die konkrete Berichterstattung trägt jedoch durch die tendenziell negative Rahmung nicht unbedingt dazu bei, das Ansehen der Institutionen und ihrer Vertreter in Deutschland zu verbessern. Den beobachteten Säkularisierungstendenzen dürfte die Berichterstattung somit eher nicht entgegenwirken, sondern könnte diesen Trend im Gegenteil sogar noch weiter verstärken.

Limitierend ist festzuhalten, dass aus forschungsökonomischen Gründen die Kirchenberichterstattung in der Tagesschau nur für sechs Jahre und zu drei Messzeitpunkten untersucht werden konnte. So können lediglich Tendenzen im Zeitverlauf aufgezeigt werden. Eine durchgängige Untersuchung der letzten Jahrzehnte wäre daher für die Zukunft wünschenswert. Dennoch machen die Ergebnisse deutlich, dass die Kirchen in der Berichterstattung entgegen den gesellschaftlichen Säkularisierungstendenzen konstant eine kleine, aber dennoch nicht zu vernachlässigende Rolle spielen und dass sich ihr Framing in den letzten 20 Jahren zu einer immer negativeren Sichtweise hin entwickelt hat, wobei besonders die katholische Kirche kritisiert wird.

Um in Zukunft verlässliche Rückschlüsse auf die Entstehungsbedingungen der Kirchenberichterstattung ziehen zu können, sind ergänzende Untersuchungen sinnvoll. Dies könnte Befragungen von Journalisten umfassen, aber auch Input-OutputAnalysen, um die Kommunikation der Kirchen (die Kommunikator-Frames) und deren Bedeutung für die Berichterstattung einzubeziehen. Außerdem müssten die Erkenntnisse über die Kirchenberichterstattung der Tagesschau in Zukunft noch stärker an die gesamte Medienberichterstattung über Kirche in Deutschland rückgebunden werden, um zu überprüfen, inwiefern die Kirchenberichterstattung einer allgemeinen Entwicklung hin zum Negativismus unterliegt. Um den etwaigen Wirkungen der Berichterstattung auf Vorstellungen, Einstellungen und Verhalten der Rezipienten nachzugehen, böten sich wiederum qualitative und quantitative Befragungsstudien an. Dabei sind auch Experimentaldesigns denkbar, die gezielt den Auswirkungen bestimmter Medien-Frames in der Berichterstattung nachgehen und z. B. in den Blick nehmen könnten, inwieweit sich diese auf die Wahrnehmung der Rezipienten der beiden Kirchen, aber auch auf die Bereitschaft zum Kircheneinund Kirchenaustritt auswirken.

Funding Open Access funding enabled and organized by Projekt DEAL.

Open Access Dieser Artikel wird unter der Creative Commons Namensnennung 4.0 International Lizenz veröffentlicht, welche die Nutzung, Vervielfältigung, Bearbeitung, Verbreitung und Wiedergabe in jeglichem Medium und Format erlaubt, sofern Sie den/die ursprünglichen Autor(en) und die Quelle ordnungsgemäß nennen, einen Link zur Creative Commons Lizenz beifügen und angeben, ob Änderungen vorgenommen wurden.

Die in diesem Artikel enthaltenen Bilder und sonstiges Drittmaterial unterliegen ebenfalls der genannten Creative Commons Lizenz, sofern sich aus der Abbildungslegende nichts anderes ergibt. Sofern das betreffende Material nicht unter der genannten Creative Commons Lizenz steht und die betreffende Handlung nicht nach gesetzlichen Vorschriften erlaubt ist, ist für die oben aufgeführten Weiterverwendungen des Materials die Einwilligung des jeweiligen Rechteinhabers einzuholen. 
Weitere Details zur Lizenz entnehmen Sie bitte der Lizenzinformation auf http://creativecommons.org/ licenses/by/4.0/deed.de.

\section{Literatur}

Bacher, G., Feichtlbauer, H., Gottlieb, S., \& Roegele, O. B. (2005). Der „Medienpapst“: Statements von Gerd Bacher, Hubert Feichtlbauer, Sigmund Gottlieb und Otto B. Roegele. Communication Socialis, 38, 281-290.

Bösch, F. (2010). Die Religion der Öffentlichkeit: Plädoyer für einen Perspektivwechsel der Kirchen- und Religionsgeschichte. Zeithistorische Forschungen, 7, 447-453.

Bruns, A. (2009). Vom Gatekeeping zum Gatewatching. Modelle der journalistischen Vermittlung im Internet. In C. Neuberger, C. Nuernbergk \& M. Rischke (Hrsg.), Journalismus im Internet. Profession. Partizipation. Technisierung (S. 107-128). Wiesbaden: VS.

Dahinden, U. (2006). Framing: Eine integrative Theorie der Massenkommunikation. Konstanz: UVK.

Dahinden, U. (2009). Die Darstellung von Religionen in Schweizer Massenmedien: Zusammenprall der Kulturen oder Förderung des Dialogs? Schlussbericht: Ein Projekt im Rahmen des Nationalen Forschungsprogramms „Religionsgemeinschaften, Staat und Gesellschaft (NFP 58)“. https://pdfs. semanticscholar.org/351b/04805f50baf1a7cdbb3d081d15c2b99c7. Zugegriffen: 27.08.2021.

Deutsche Bischofskonferenz - DBK (2020). Katholische Kirche in Deutschland: Zahlen und Fakten 2019/20. https://www.dbk.de/fileadmin/redaktion/Zahlen\%20und\%20Fakten/Kirchliche\%20Statistik/ Allgemein_-_Zahlen_und_Fakten/AH-315-ZuF_2019-2020_Ansicht.pdf. Zugegriffen: 27.08.2021.

Die Medienanstalten (2020). Medienstaatsvertrag (MStV) vom 14./28. April 2020 in Kraft seit 7. November 2020. https://www.die-medienanstalten.de/fileadmin/user_upload/Rechtsgrundlagen/Gesetze_ Staatsvertraege/Medienstaatsvertrag_MstV.pdf. Zugegriffen: 27.08.2021.

Donsbach, W. (1987). Journalismusforschung in der Bundesrepublik: Offene Fragen trotz „Forschungsboom“. In J. Wilke (Hrsg.), Zwischenbilanz der Journalistenausbildung (S. 105-144). München: Ö1schläger Verlag.

Eilders, C. (1997). Nachrichtenfaktoren und Rezeption. Eine empirische Analyse zur Auswahl und Verarbeitung politischer Information. Opladen: Westdeutscher Verlag.

Entman, R. M. (1993). Framing: toward clarification of a fractured paradigm. Journal of Communication, 43(4), 51-58.

Esser, F. (1999). 'Tabloidization' of news: a comparative analysis of Anglo-American and German press journalism. European Journal of Communication, 14, 291-324.

Evangelische Kirche in Deutschland - EKD (2014). Engagement und Indifferenz: Kirchenmitgliedschaft als soziale Praxis: V. EKD-Erhebung über Kirchenmitgliedschaft. https://www.ekd.de/ekd_de/ds_ doc/ekd_v_kmu2014.pdf. Zugegriffen: 27.08.2021.

Evangelische Kirche in Deutschland - EKD (2021). Gezählt 2021: Zahlen und Fakten zum kirchlichen Leben. https://www.ekd.de/ekd_de/ds_doc/Gezaehlt_zahlen_und_fakten_2021.pdf. Zugegriffen: 27.08.2021.

Favre, V. (2011). Resultate der quantitativen Inhaltsanalyse: Religion(en). In I. C. fünf Schweizer Fernsehprogrammen \& Jecker (Hrsg.), Religionen im Fernsehen: Analysen und Perspektiven (S. 65-98). Konstanz: UVK.

Forschungskonsortium, W. J. T. (2007). Megaparty Glaubensfest: Weltjugendtag: Erlebnis - Medien - Organisation. Wiesbaden: VS.

Galtung, J., \& Ruge, M.H. (1965). The structure of foreign news. The presentation of the Congo, Cuba and Cyprus crises in four Norwegian newspapers. Journal of Peace Research, 2, 259-298.

Gottschlich, M. (1995). Was ,glauben“ Österreichs Journalisten? Untersuchung über die religiöse Einstellung und Werteorientierung der Medien-Elite. Communicatio Socialis, 28, 303-320.

Günther, W. (2000). Zur Entwicklung der religiösen Kultur: Eine Sekundäranalyse von Daten des Allensbacher Archivs. https://www.ifd-allensbach.de/studien-und-berichte/veroeffentlichte-studien.html? tx_studies_studieslist $\% 5$ Baction $\% 5 \mathrm{D}=$ search\&tx_studies_studieslist $\% 5 \mathrm{Bcontroler} \% 5 \mathrm{D}=\mathrm{Resarch} \&$ cHash=94ec651a685695dad801404a73658c9. Zugegriffen: 10.03.2020.

Hagen, L. M. (1992). Die opportunen Zeugen. Konstruktionsmechanismen von Bias in der Zeitungsberichterstattung über die Volkszählungsdiskussion. Publizistik, 37, 444-460.

Hempelmann, H., \& Flaig, B. B. (2019). Aufbruch in die Lebenswelten: Die zehn Sinus-Milieus als Zielgruppen kirchlichen Handelns. Wiesbaden: Springer VS. 
Hepp, A., \& Krönert, V. (2009). Medien - Event - Religion: Die Mediatisierung des Religiösen. Wiesbaden: VS.

Kepplinger, H.M. (1989). Theorien der Nachrichtenauswahl als Theorien der Realität. Aus Politik und Zeitgeschickte, 15, 3-16.

Kepplinger, H. M. (1998). Der Nachrichtenwert der Nachrichtenfaktoren. In C. Holtz-Bacha, H. Scherer \& N. Waldmann (Hrsg.), Wie die Medien die Welt erschaffen und wie die Menschen darin leben (S. 19-38). Opladen: Westdeutscher Verlag.

Kerr, P. A. (2009). The framing of fundamental christians: network television news, 1980-2000. Journal of Media and Religion, 2, 203-235.

Kerr, P. A., \& Moy, P. (2002). Newspaper coverage of fundamentalist christians, 1980-2000. Journalism and Mass Communication Quarterly, 79(19), 54-72.

Klein, M.W., \& Maccoby, N. (1954). Newspaper objectivity in the 1952 campaign. Journalism Bulletin, 31, 285-296.

Klenk, C. (2006). Hauptsache Papst, Hauptsache Emotion: Der Kölner Weltjugendtag in der Presse. Communication Socialis, 39, 337-360.

Klenk, C. (2008). Ein deutscher Papst wird Medienstar: Benedikt XVI. und der Kölner Weltjugendtag in der Presse. Berlin: LIT.

Knott, K., Poole, E., \& Taira, T. (2013a). Christianity, secularism and religious diversity in the British media. In M. Gillespie, D. E. J. Herbert \& A. Greenhill (Hrsg.), Social media and religious change (S. 37-58). Berlin: De Gruyter.

Knott, K., Poole, E., \& Taira, T. (2013b). Media portrayals of religion and the secular sacred: representation and change. New York: Routledge.

Koch, C. (2009). Das Politische dominiert: Wie Schweizer Medien über Religionen berichten. Communication Socialis, 42, 365-381.

Koch, C. (2012). Religion in den Medien. Eine quantitative Inhaltsanalyse von Medien in der Schweiz. Konstanz: UVK.

Kolmer, C. (2008). Nachrichtenfaktor Prominenz hält Kirche in den Schlagzeilen: Analyse von Fernsehnachrichten 2001 bis 2008. Communication Socialis, 41, 412-416.

Kolmer, C. (2014). Kirche in den Medien: Mediatisierung, Personalisierung, Skandalisierung: Eine Langzeitanalyse zeigt, wie die Kirche in den Jahren 2001 bis 2013 im Fernsehen dargestellt wurde. Communicatio Socialis, 47, 244-249.

Krüger, U. M., \& Zapf-Schramm, T. (2019). InfoMonitor 2018: GroKo und Migrationsdebatte prägten die Fernsehnachrichten: Analyse der Nachrichtensendungen von Das Erste, ZDF; RTL und Sat.1. Media Perspektiven, 50(2), 44-73.

Kunczik, M., \& Zipfel, A. (2005). Publizistik: Ein Studienhandbuch. Köln: Böhlau.

Landmeier, C., \& Daschmann, G. (2011). Im Seichten kann man nicht ertrinken? Boulevardisierung in der überregionalen deutschen Qualitätspresse. In R. Blum, H. Bonfadelli, K. Imhof \& O. Jarren (Hrsg.), Krise der Leuchttürme öffentlicher Kommunikation: Vergangenheit und Zukunft der Qualitätsmedien (S. 177-191). Wiesbaden: VS.

Leidenberger, J. (2015). Boulevardisierung von Fernsehnachrichten: Eine Inhaltsanalyse deutscher und französischer Hauptnachrichtensendungen. Wiesbaden: Springer VS.

Link, C. (1995a). Der Anspruch der Kirchen auf Präsenz in den öffentlich-rechtlichen und privat-rechtlichen Massenmedien des Rundfunks und des Fernsehens. In E. Friesenhahn \& J. Listl (Hrsg.), Handbuch des Staatskirchenrechts der Bundesrepublik Deutschland (S. 251-284). Berlin: Duncker \& Humblot.

Link, C. (1995b). Die gesetzlichen Regelungen der Mitwirkung der Kirche in den Einrichtungen des Rundfunks und Fernsehens. In E. Friesenhahn \& J. Listl (Hrsg.), Handbuch des Staatskirchenrechts der Bundesrepublik Deutschland (S. 285-303). Berlin: Duncker \& Humblot.

Matthes, J. (2007). Framing-Effekte. Zum Einfluss der Politikberichterstattung auf die Einstellung der Rezipienten. München: Fischer.

Matthes, J. (2014). Framing. Baden-Baden: Nomos.

Maurer, M., \& Reinemann, C. (2006). Medieninhalte. Eine Einführung. Wiesbaden: VS.

Maurer, T., Wagner, M., \& Weiß, H.-J. (2020). Fernsehnachrichten: Mehr als Klimawandel, Brexit, Europaund Landtagswahlen: Ergebnisse des Nachrichtenmonitors 2019. Media Perspektiven, 51(2), 62-86.

McCombs, M., \& Reynolds, A. (2009). How the news shapes our civic agenda. In J. Bryant \& M. B. Oliver (Hrsg.), Media effects. Advances in theory and research (S. 1-16). New York: Routledge.

McCombs, M.E., \& Shaw, D.L. (1972). The agenda-setting function of mass media. Public Opinion Quarterly, 36, 176-187. 
McCombs, M.E., \& Shaw, D. L. (1993). The evolution of agenda-setting research: twenty-five years in the marketplace of ideas. Journal of Communication, 43(2), 58-67.

Meier, D. (2006). Kirche in der Tagespresse: Empirische Analyse der journalistischen Wahrnehmung von Kirche anhand ausgewählter Zeitungen. Erlangen: Christliche Publizistik.

NDR-StV (2005) = NDR-Staatsvertrag idF vom 17./18. Dez. 1991 (HambGVB1 1992, S. 39), zuletzt geändert am 1./2. Mai 2005 (HambGVB1 2005, S. 263). Zugegriffen: 27.08.2021.

Norddeutscher Rundfunk NDR (2019). Welche Aufgaben hat der NDR in der ARD? https://www.ndr.de/ der_ndr/zahlen_und_daten/Welche-Aufgaben-hat-der-NDR-in-der-ARD, ardaufgaben100.html. Zugegriffen: 27.08.2021.

Pickel, G. (2017). Religiosität in Deutschland und Europa - Religiöse Pluralisierung und Säkularisierung auf soziokulturell variierenden Pfaden. Zeitschrift für Religion, Gesellschaft und Politik, 1, 37-74.

Pollack, D. (2016). Wiederkehr der Religion oder Rückgang ihrer Bedeutung: Religiöser Wandel in Westdeutschland. Soziale Passagen, 8(1), 5-28.

Potthoff, M. (2012). Medien-Frames und ihre Entstehung. Wiesbaden: Springer.

Robinson, G. J. (1973). Fünfundzwanzig Jahre „Gatekeeper“-Forschung. Eine kritische Rückschau und Bewertung. In J. Aufermann, H. Bohrmann \& R. Sülzer (Hrsg.), Gesellschaftliche Kommunikation und Information. Forschungsrichtungen und Problemstellungen. Ein Arbeitsbuch zur Massenkommunikation (Bd. 1, S. 344-355). Frankfurt am Main: Athenäum.

Rosengren, K. E. (1979). Bias in news: methods and concepts. Studies of Broadcasting, 15(5), 31-45.

Roth, A. (2004). Das Bild von Religion und Kirche bei ostdeutschen Zeitungsredakteuren. Communicatio Socialis, 37, 329-347.

Schäfer, M. (2018). Medienhype „Hirndoping“? Die Rolle der Journalisten in der gesellschaftlichen Debatte um Neuroenhancement. Baden-Baden: Nomos.

Scheufele, B. (2003). Frames - Framing - Framing-Effekte. Theoretische und methodische Grundlegung des Framing-Ansatzes sowie empirische Befunde zur Nachrichtenproduktion. Wiesbaden: Westdeutscher Verlag.

Scheufele, D. A. (1999). Framing as a theory of media effects. Journal of Communication, 49(1), 103-122.

Schielicke, A.-M. (2014). Rückkehr der Religion in den öffentlichen Raum? Kirche und Religion in der deutschen Tagespresse von 1993 bis 2009. Wiesbaden: Springer.

Schönbach, K. (1977). Trennung von Nachricht und Meinung. Empirische Untersuchung eines journalistischen Qualitätskriteriums. Freiburg: Alber.

Schulz, W. (1976). Die Konstruktion von Realität in den Nachrichtenmedien. Analyse der aktuellen Berichterstattung. Freiburg: Alber.

Semetko, H.A., \& Valkenburg, P. M. (2000). Framing European politics. A content analysis of press and television news. Journal of Communication, 50(2), 93-109.

Staab, J. F. (1990). Nachrichtenwert-Theorie. Formale Struktur und empirischer Gehalt. Freiburg: Alber.

Underwood, D. (2002). From Jahweh to Yahoo! The religious roots of the secular press. Urbana: University of Illinois Press.

Vertrag 2006 = Vertrag zwischen dem Heiligen Stuhl und der Freien und Hansestadt Hamburg idF vom 6. 7. 2006 (HmbGVB1 2006, S. 435). Zugegriffen: 27.08.2021.

Weischenberg, S. (1992). Journalistik: Theorie und Praxis aktueller Medienkommunikation. Opladen: Westdeutscher Verlag.

Weischenberg, S., Malik, M., \& Scholl, A. (2006). Die Souffleure der Mediengesellschaft: Report über Journalisten in Deutschland. Konstanz: UVK.

Wernicke, C., \& Zoch, A. (2021). Köln ist überall. Süddeutsche Zeitung. https://www.sueddeutsche.de/ politik/kirche-missbrauch-koeln-1.5216918. Zugegriffen: 27.08.2021.

White, D. M. (1950). The "Gate Keeper". A case study in the selection of news. Journalism Quarterly, 27, 383-390.

Willenberg, R. (2001). Rundfunk in kirchlicher Trägerschaft. Frankfurt a. M.: Peter Lang.

Hannah Thielmann ist wissenschaftliche Hilfskraft am Institut für Publizistik der Johannes GutenbergUniversität Mainz und studiert Erziehungswissenschaft (M.A.) am Institut für Erziehungswissenschaft der Johannes Gutenberg-Universität Mainz.

Dr. Markus Schäfer ist wissenschaftlicher Mitarbeiter am Institut für Publizistik der Johannes GutenbergUniversität Mainz. 